\title{
A novel temperature controller for in-situ measurement of radiation-induced changes in temperature effects on space electronics
}

\author{
Jiri Haze, Jiri Hofman*
}

\begin{abstract}
The paper discusses a novel temperature controller and a related test method allowing in-situ measurement of total ionising dose-induced changes in the impact of temperature on electronic devices for space applications. Various results of pilot radiation experiments (testing commercial PMOS transistors, RADFETs, and voltage references) are also presented.

K e y w or d s: automated test equipment, thermoelectric cooler, thermometers, test software, test methods, temperature effects, RADFET, TID, PMOS, temperature coefficients, MTC
\end{abstract}

\section{Introduction}

Over the decades, the parameters measured during ionising dose tests (TIDs) were mainly used to determine problems associated with degradation of the reliability of electronic components $[1,2]$. The relevant research conducted at Brno University of Technology (BUT) is focused on a different approach: testing and modelling of TID-induced degradation of the performance of electronic systems, data acquisition ones in particular. The resulting models may be utilised for computing TID-induced reduction in the measurement accuracy (uncertainty) of high resolution space data acquisition systems (eg scientific experiments on board space missions). A key source of measurement errors is ambient temperature variation; thus, the measurement accuracy modelling must take into account the temperature coefficients (TCs) of the accuracyrelated parameters.

Only a few papers have been published to date on radiation-induced TC changes [3-5]; according to [3], a clear impact of radiation on a RADFET TC was observed (Fig. 1, adopted from [3]). The devices were exposed in the ZB mode. Data originate from tests using $X$-ray and a mixed hadron field (CERN-IRRAD6). During the reported experiments, a standard bench testing procedure was employed: the devices under test (DUTs) were exposed to a certain dose at ambient temperature, and then a long-duration temperature sweep in a temperature chamber was performed to observe the TC of the measured parameters. Such a method is relatively simple but also exhibits several disadvantages, as follows: 1) the irradiated devices can be thermally annealed during the TC measurement, and the radiation-induced changes may become lost (or suppressed) due to the long-duration exposure to an elevated temperature; 2) the number of TC measurements is limited (practically to only a few [6]); 3 ) the repeatability of the measurements is restricted by the accuracy of placing the samples in the chamber, the timing accuracy of the temperature profile, and other factors. As the TCs of modern devices are very low, this standard approach may not be sufficiently sensitive.

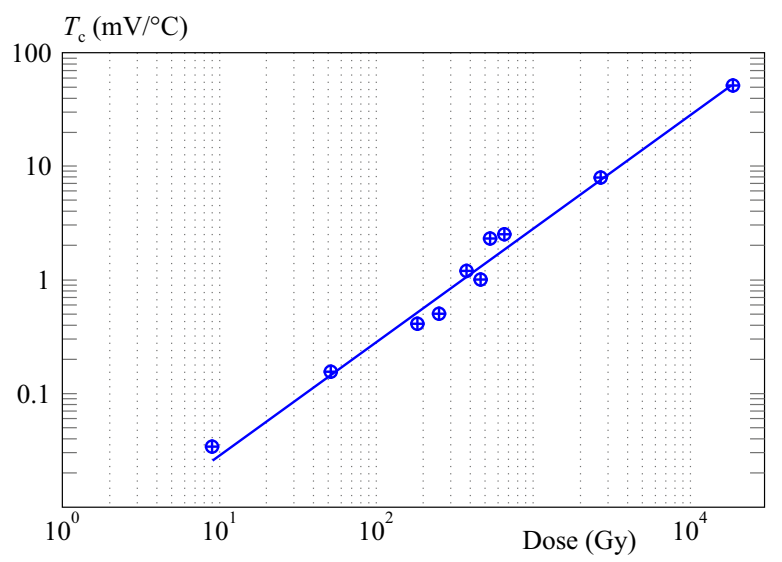

Fig. 1. The impact of radiation on the temperature coefficient (TC) measured at $I_{d}=160 \mu \mathrm{A}$, for an irradiated RadFET REM TOT$501 \mathrm{C}$ type $\mathrm{K}\left(t_{\mathrm{ox}}=0.25 \mu \mathrm{m}\right)$

We describe a novel in-situ test method and a temperature controller developed to suppress the drawbacks of the standard procedure by measuring the TCs during uninterrupted irradiation (in-situ). The technique is designed for use in TID experiments with the following devices:

- RADFETs/PMOS - the TC is one of the key measurement errors in dosimetry applications. The experiments will help to improve the understanding of the issue and to develop a more precise RADFET calibration procedure.

- Voltage references - the output voltage TC is a critical parameter for any precision application of a voltage reference, especially for data acquisition systems.

- A/D converters - most conversion errors are temperature-sensitive; the goal is to obtain data for at least

* Brno University of Technology, Technicka 8, Brno, Czech Republic, haze@feec.vutbr.cz 


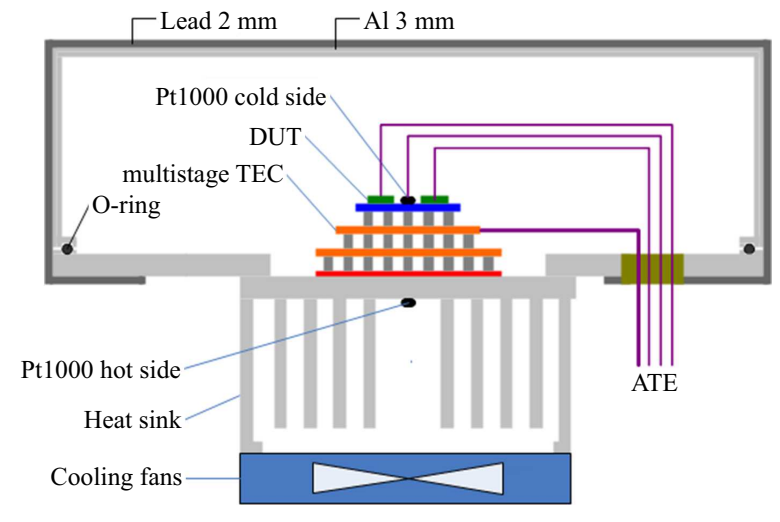

Fig. 2. The mechanical structure of the TEC assembly placed in the DUT container

the TC of gain and offset as well as the linearity errors in selected $\mathrm{A} / \mathrm{D}$ converters.

As the first step, the temperature coefficients of commercial PMOS transistors were investigated within a proof-of-concept experiment [7]. This allowed the methodology to be further developed and refined, before being tested with more complex devices (RADFETs [8] and precision voltage references [9]).

\section{In-situ TID-TC test method}

The key technical challenge associated with in-situ temperature coefficient measurements is to expose the DUTs to a temperature sweep during an irradiation cycle. Typically, standard bench TID tests are employed to simulate the full temperature ranges of the devices, using temperature chambers such as those by Weiss Technik [10]. This approach is relatively simple but exhibits multiple serious constraints, including the following ones:

- The irradiated devices can be thermally annealed during the TC measurement, and the radiation-induced changes may become lost due to the long-duration exposure to an elevated temperature.

- The temperature profile of a large chamber might be too slow; thus, the two-hour test window might not be followed [11].

- The repeatability of the measurements is limited by the accuracy of placing the samples in the chamber and the timing accuracy of the temperature profile.

- Temperature chambers are usually large and expensive, which is inconvenient especially for low-cost testing of COTS (Commercial of the Shelf) components.

Modern localised temperature test systems (LTTS) were designed to address the constraints of traditional testing in temperature chambers. These advanced instruments typically consist of a thermal head, which controls the temperature of the DUTs, and a base unit generating air of stable temperature [12]. During a test cycle, the head is attached to the PCB, and the forced air stream ensures the heating or cooling of an individual DUT. The LTTS concept is ideal for bench testing, as it allows fast temperature changes and secures good accuracy/repeatability.

For the in-situ tests, none of the methods described above is suitable. Convenient temperature chambers are too large to be accommodated by an irradiation facility, and the LTTS contains electronics that would require radiation hardening. In both cases, the prices of the instruments are not affordable by low-cost projects.

A novel technique for fine DUT temperature control was developed. As is shown in Fig. 2, the method exploits the thermoelectric cooler technology (TEC). The DUTs were attached to the cold side of the applied multistage TEC. The cooling of the TECs hot side was ensured by a powerful heat sink with attached fans. The temperatures of the DUTs as well as the TECs hot side were monitored by using radiation tolerant Pt1000 sensors.

In the proposed approach, the DUTs are attached to the cold side of the employed multistage TEC by utilizing thermally conductive compounds or glues. The hot side of the TEC is cooled by a powerful heat sink, with the ventilation ensured via an external AC blower. The temperatures of the DUTs and the TECs hot side are monitored using radiation tolerant Pt1000 sensors. In both the cooling and the heating mode, the TEC can be operated by changing the polarity of the DC current [13]. The TEC assembly must be placed in a hermetic container to minimise the humidity of the air around the TECs cold side and thus to suppress frost build-up.

\section{In-situ DUT temperature controller}

A novel DUT temperature controller (DTC) had to be developed and tested to support the in-situ TID-TC experiments, as defined in the previous section. The DTC system was designed with respect to meeting the following requirements:

- DUT temperature range: -40 to $100{ }^{\circ} \mathrm{C}$ (the industrial range of COTS ICs), optionally -55 to $125^{\circ} \mathrm{C}$ (the military range).

- Temperature control stability: $0.05^{\circ} \mathrm{C}$ (to suppress the TC-induced noise of the DUT output voltage to $1 \mathrm{ppm}$ for $T_{C}=20 \mathrm{ppm} /{ }^{\circ} \mathrm{C} .1 \mathrm{ppm}$ is the typical resolution of DMMs).

- Temperature measurement accuracy: $<0.5^{\circ} \mathrm{C}$ (for the entire range).

- Fast temperature setting/settling: < 100 s (to accelerate the TID testing).

- Arbitrary temperature profiles to be generated by STS.

- Ambient temperature: 15 to $35^{\circ} \mathrm{C}$ (both the DTC system and the irradiation container).

- EMC/EMI: minimum noise induced to the DUTs from the TEC. 


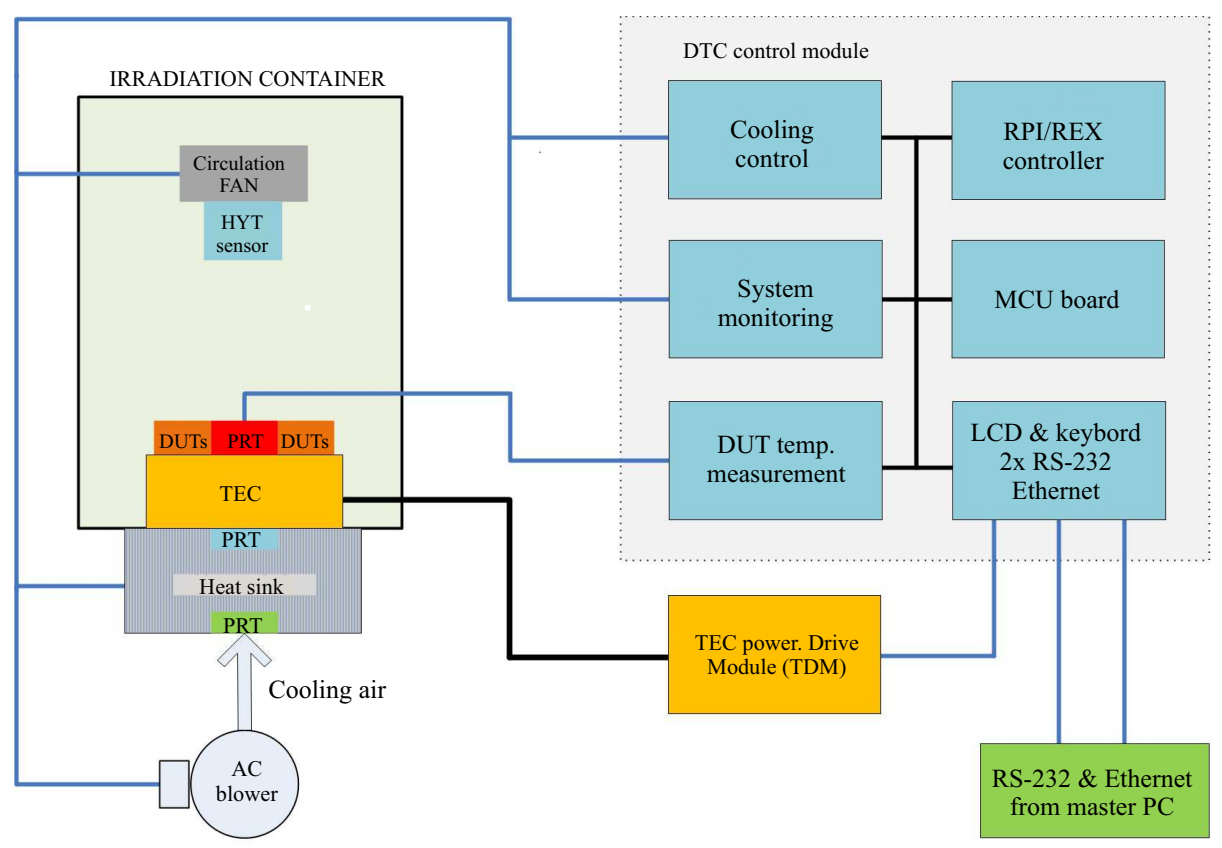

Fig. 3. The block diagram of the DUT temperature control system

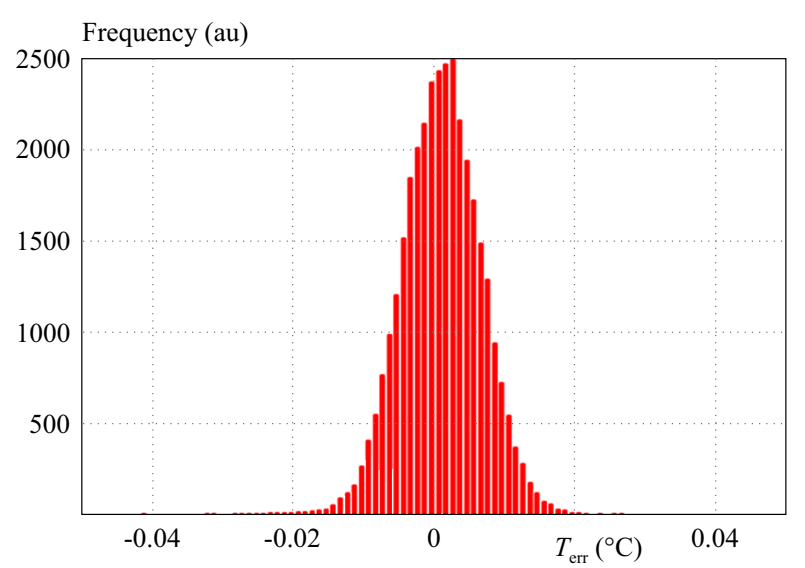

Fig. 4. The histogram of the static test

- The components in the irradiation container are to be TID-tolerant up to $150 \mathrm{krad}(\mathrm{Si})$.

The DTC system consisted of three principal elements, as displayed in Fig. 3. The TEC assembly was an integrated part of the mechanical structure of the irradiation container. The setup comprised the TEC assembly installed in the irradiation container as well as the DTC and TDM modules placed in the test equipment area (outside the irradiation facility) in-situ DUT temperature controller. The DTC control module contained all the measuring electronics and control features. The TEC was powered by a TEC drive module (TDM), and the DTC communicated with the TDM via RS-232. The commands and data from/to the master PC were transferred via RS-232 and Ethernet buses.

Precise, low noise DUT temperature measurement was an essential capability of the DTC system. The platinum resistance thermometer (PRT) sensing technology was chosen based on its excellent accuracy, long-term stability, and standardised temperature response [14]. Moreover, ceramics-based PRTs were found to be inherently radiation tolerant $[2,15]$; their resistance was measured with a Texas Instruments ADS1248 $\Delta \Sigma \mathrm{A} / \mathrm{D}$ converter [16]. The ADS1248 not only ensured high-performance A/D conversion but also provided programmable excitation current sources to drive the PRTs.

In order to control the temperature of the DUTs, a powerful thermoelectric cooler (TEC) was used to allow stable and precise temperature control, including fast changes of the DUT temperature. The radiation response of a similar TEC was measured, with no obvious degradation observed [17].

An advanced PID algorithm was running a Linuxbased microcontroller module [18]. The development of the actual TEC power drive module had been expected to require complex efforts; thus, a decision was made to reuse an existing MPPL design adopted from a previous research programme [19].

The MPPL (Maximum Power Point Load) had originally been developed for I-V characterisation of photovoltaic panels, embodying a programmable DC load with the current range of $10 \mathrm{~A}$ and $0.001 \%$ resolution. The MPPL design exploited a single high-power MOS transistor driven by an analogue PI controller. The setpoint voltage for the PI controller was generated by a 20-bit DAC controlled by an MCU. The conversion of the MPPL to the TDM involved adding a DC power supply, extending the hardware with a relay-based TEC polarity control board, and developing new MCU software.

\section{Testing of the DTC system}

The DTC system was tested at all stages of the development process, typically on the subsystem level. Series 
Measured temperature $\left({ }^{\circ} \mathrm{C}\right)$

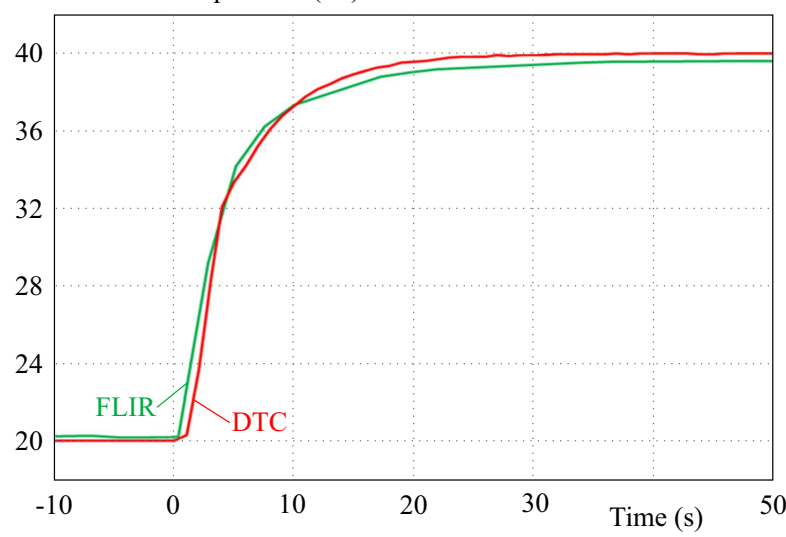

Fig. 5. The dynamic test of the DTC system; the chart represents the data obtained from the DTC and the FLIR camera

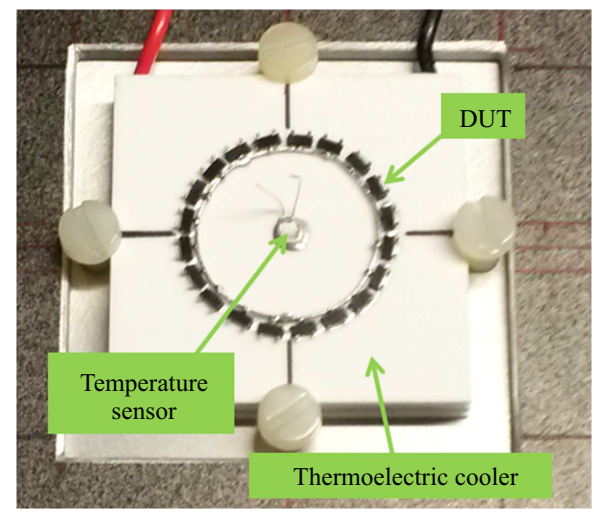

Fig. 6. The inside of the DUT container prior to completion of the hardware assembly and final sealing

of tests to measure the performance of the system and to validate its functionality were performed. These included the following tasks and procedures:

- Tuning the PID controller to reach optimum static and dynamic performance.

- Testing the protection system for various failure modes and their combinations, including communication errors.

- A static test (temperature control stability).

- A dynamic test (response to a temperature setpoint change).

- A test of the DUT temperature measurement accuracy.

- A cooling capability test (focused on the impact of the ambient air temperature).

- Inner atmosphere measurement.

The static test was performed at multiple stages of the actual development; however, the most significant step consisted in the final test, during which the relevant software recorded the DUT temperatures in a fully assembled experiment. The result of the static test during the preirradiation phase of the pilot PMOS experiment is shown in Fig. 4. The histogram was constructed from 34,000 random samples obtained through two-day recording. The

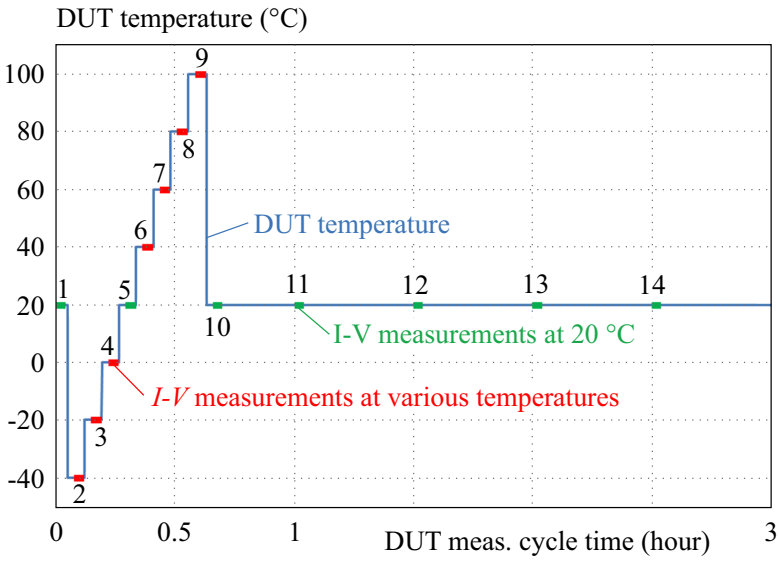

Fig. 7. The timing of the DUT measurement cycle, including the temperature profile

DTC was commanded to maintain the DUT temperature at $20^{\circ} \mathrm{C}$. While $94 \%$ of the measurements lay in the $\pm 0.01{ }^{\circ} \mathrm{C}$ window, $99.8 \%$ were within $\pm 0.02{ }^{\circ} \mathrm{C}$.

The estimated uncertainty of the DUT temperature measurements corresponded to $0.36{ }^{\circ} \mathrm{C}\left(k=2,100{ }^{\circ} \mathrm{C}\right.$ range). This value could not be verified by a proper test in a calibrated temperature oven due to budget limits; however, an electrical calibration procedure (calibration of the PRT resistance measurement) was performed before each experiment. After the DTC system had been in practical use for two years, the original calibration was verified with a high-end multimeter, a DMM7510 [20]. This independent test showed that, during the time, the DUT measurement system had drifted by less than $0.1^{\circ} \mathrm{C}$; the observation was in good agreement with the MU estimation related to the electronics of the DUT temperature measurements.

The dynamic testing was performed by acquiring the DTC data of the DUT temperatures and by measuring the temperature of a DUT (an 8-lead SOIC package attached to the TEC) with a high-resolution FLIR thermal camera [21]. The measured DUT temperature step changes between 20 and $40^{\circ} \mathrm{C}$ are indicated in Fig. 5. At time $t=0 \mathrm{~s}$, the DTC was commanded to set the value to $40{ }^{\circ} \mathrm{C}$, and it took $30 \mathrm{~s}$ for the DTC-measured temperature to stabilise. However, the FLIR measurements of the DUT package showed that the DUTs surface temperature had stabilised at $t=50 \mathrm{~s}$. This experiment proved (through the use of a different sensing technology) that the dynamic parameters of the DTC systems exceed the requirements of the planned experiments.

\section{PMOS TID-TC experiment}

The pilot experiment was focused on testing COTS PMOS transistors [7]. A set of twenty low power, ZVP132 0FPA PMOS transistors was used [22]. To measure the bias sensitivity of the threshold voltage shift and the TCs, the DUTs were separated into five bias groups: $0 \mathrm{~V}$ (all pins grounded), $3 \mathrm{~V}, 5 \mathrm{~V}, 12 \mathrm{~V}$, and $18 \mathrm{~V}$. Each of the groups contained four devices. 
Threshold voltage shift $\Delta V_{\mathrm{T}}(\mathrm{V})$

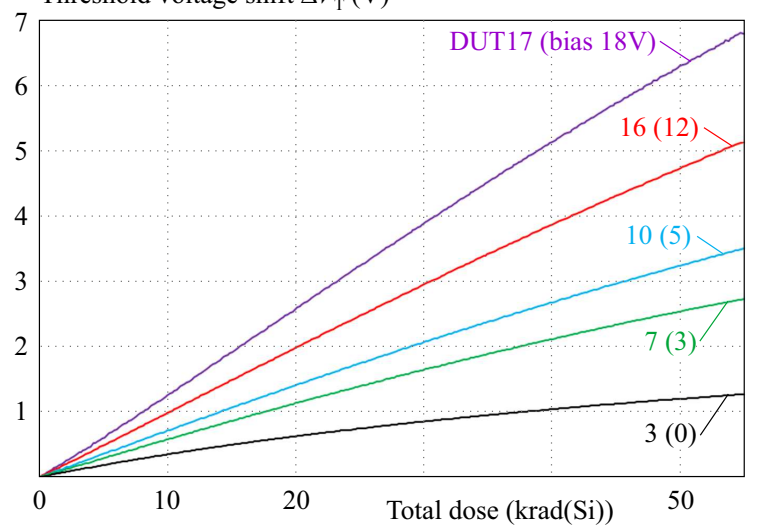

Fig. 8. The experiment 1 threshold voltage shift $\Delta V_{\mathrm{T}}$ at the drain current of $1 \mathrm{~mA}$ plotted as a function of the total dose for the selected DUTs

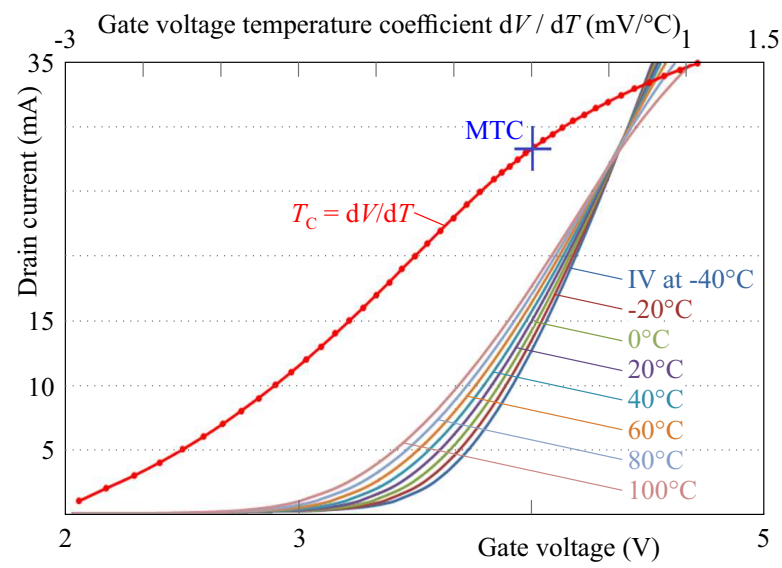

Fig. 10. The temperature sweep $I-V$ measurements for DUT01 prior to the irradiation within experiment 1

The DUTs were mounted on the TEC surface in a circle, by using thermally conductive glue. The assembly is illustrated in Fig. 6.

A fully automated, PC-based system facilitated the DUT measurements during the experiment; the setup comprised a DUT container placed in an irradiation cell and a set of test instruments located in a nearby test laboratory. All the measurements relied on a Keithley 2611B Source Measurement Unit (SMU) combined with custom made electronics, including relay drivers and programmable DUT bias voltage sources. The DUT temperature controller operated independently (but synchronously with the PC).

For most of the irradiation time, the DUTs were in the "exposure" mode, meaning that the drain (D) and source $(\mathrm{S})$ were grounded, with the gate $(\mathrm{G})$ connected to the relevant bias voltage source.

Regular $I-V$ curve measurements were carried out, as represented in Fig. 7. The DUT $I-V$ curves were measured at $20^{\circ} \mathrm{C}$ every 30 minutes during the idle time (green dots) and at various temperatures in the course of the temperature sweep (red dots). The temperature sweep starts with the DUT cool down mode and continues with

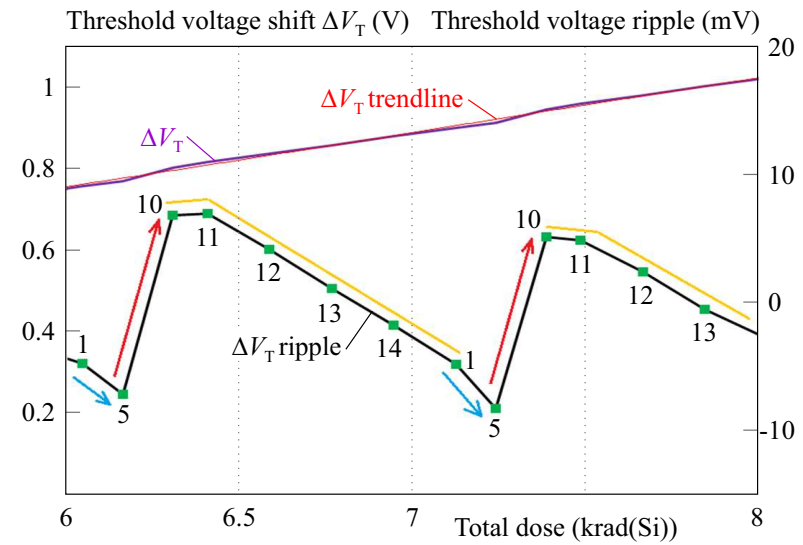

Fig. 9. A detail of the experiment 1 DUT17 recorded over two DUT measurement cycles

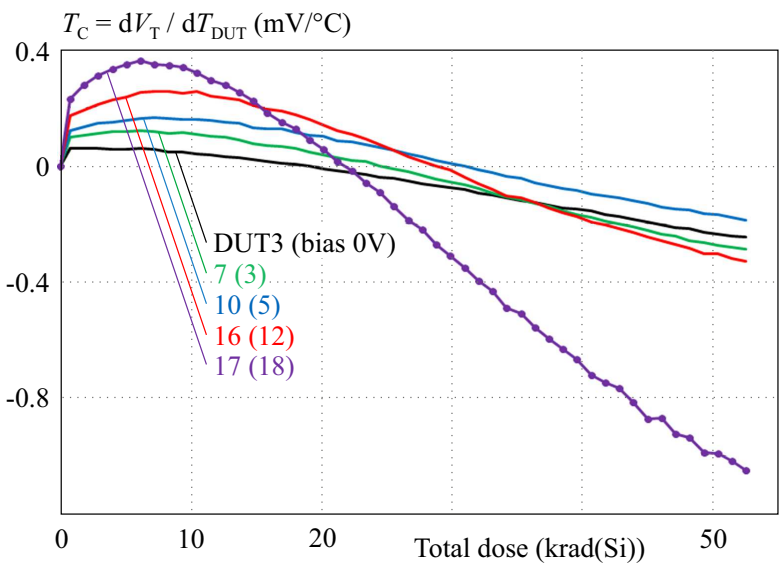

Fig. 11. The experiment 1 TID-induced changes of the $V_{\mathrm{T}} \mathrm{TC}$ at the drain current of $1 \mathrm{~mA}$

the warm-up one. Notable is the $I-V$ measurement at the idle temperature between these two modes (No. 5).

The DUTs were subsequently switched from exposure to "reader" mode, during which G and D were grounded, $\mathrm{S}$ was driven with a constant current from the SMU, and the S-D voltage (DUT threshold voltage $V_{T}$ ) was measured. Sixty current steps were evaluated between $10 \mu \mathrm{A}$ and $35 \mathrm{~mA}$. The DUT multiplexing was performed via mechanical relays placed in the DUT container near the DUTs. The measurement of one $I-V$ curve took 7.5 seconds and, compared to [4] and [5], there were no additional delays between setting the drain current and measuring the VT. Such a fast measurement process enabled us to reduce the DUTs exposure time to elevated temperatures. The dose rate of $360 \mathrm{rad}(\mathrm{Si}) / \mathrm{hr}$ (or $100 \mathrm{mrad}(\mathrm{Si}) / \mathrm{sec}$ ) was used. The cobalt-60 source and the irradiation position were calibrated using a $0.125 \mathrm{~cm}^{3}$ PTW farmer ion chamber placed inside the DUT container instead of the DUTs.

As expected, a TID-induced threshold voltage shift $\Delta \mathrm{V}_{\mathrm{T}}$ was observed in all the DUTs. An isothermal record of the most sensitive DUTs is displayed in Fig. 8. Strong bias voltage dependence is indicated, together with a 


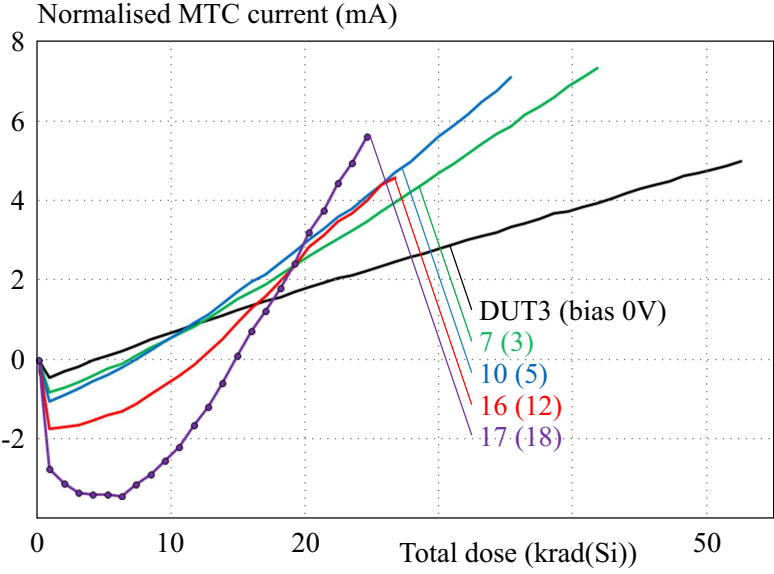

Fig. 12. The experiment 1 TID-induced changes of the MTC points in selected DUTs

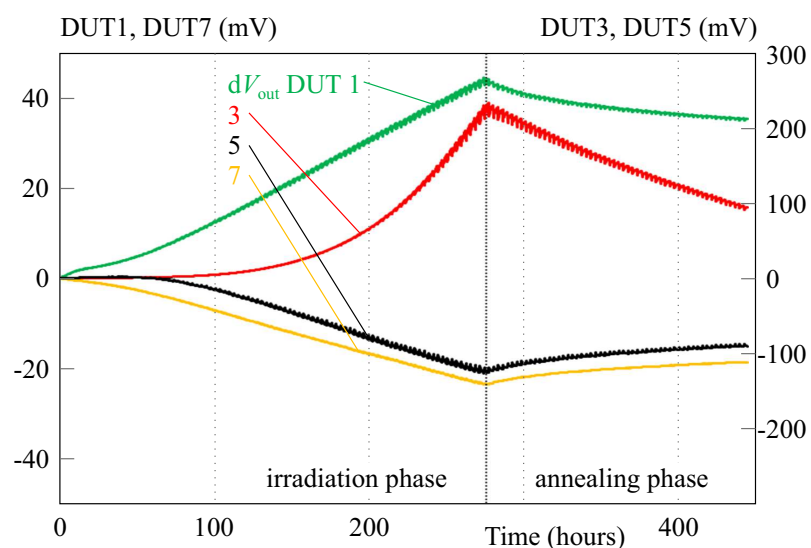

Fig. 13. The output voltage degradation and recovery in the unbiased DUTs during the irradiation and annealing

Table 1.

\begin{tabular}{|c|c|c|c|c|c|}
\hline $\begin{array}{l}\text { Part number } \\
\text { Device marking }\end{array}$ & $\begin{array}{c}\text { Unbiased } \\
\text { DUT }\end{array}$ & $\begin{array}{c}\text { Biased } \\
\text { DUT }\end{array}$ & $\begin{array}{c}\text { VOUT } \\
(\mathrm{V})\end{array}$ & $\begin{array}{c}\text { TC typ. } / \max \\
\left(\mathrm{ppm} /{ }^{\circ} \mathrm{C}\right)\end{array}$ & $\begin{array}{c}\text { Reference } \\
\text { circuit }\end{array}$ \\
\hline LT1236BIS8-5, 443-e3/N74-928 & DUT1 & DUT2 & 5.000 & $5 / 10$ & buried Zener diode \\
\hline ADR440ARZ, \#006-0328 1800328 & DUT3 & DUT4 & 2.048 & $2 / 10$ & XFET \\
\hline LT1460EIS8-2.5, 434-e3/N74-928 & DUT5 & DUT6 & 2.500 & $10 / 20$ & bandgap \\
\hline ADR03ARZ, \#420-2685 2882685 & DUT7 & DUT8 & 2.500 & $3 / 10$ & bandgap \\
\hline
\end{tabular}

small ripple on the curves. All the measurements plotted were taken at the idle temperature of $20^{\circ} \mathrm{C}$. The threshold voltage shift $\Delta \mathrm{V}_{\mathrm{T}}$ is a sensitive measure of the oxide charge growth.

The plotted $\Delta \mathrm{V}_{\mathrm{T}}$ curves are not smooth, exhibiting a small but consistent ripple in each DUT. This condition was one of the problems anticipated, as the DUT temperature had been cycled regularly during the irradiation. To analyse the issue, the ripple was "demodulated" from the $\Delta \mathrm{V}_{\mathrm{T}}$ curves and examined with respect to the timing and DUT temperature profile of the measurement cycle (Fig. 9 versus Fig. 7). The resulting plot shows that the ripple has an obvious positive temperature dependence. The DUT The temperature sweeps significantly altered the $\Delta V_{\mathrm{T}}$ values. The $\Delta V_{\mathrm{T}}$ curve was fitted with a polynomial function, and the resulting trend-line was subtracted from the original $\Delta V_{\mathrm{T}}$ curve to obtain the relevant ripple(black curve). The $I-V$ measurement numbers and DUT temperature trends were added to indicate the influence of the DUT temperature sweep on the isothermal measurements of $\Delta \mathrm{V}_{\mathrm{T}}$.

As shown in Fig. 7 ( $I-V 2$ to 9 ), the temperature sweep $I-V$ curves were measured every three hours, representing the accumulated dose of $1.08 \mathrm{krad}(\mathrm{Si})$ per measurement cycle. In total, we employed seven temperature steps to measure (a) - the TC of the DUT threshold voltage $\mathrm{V}_{\mathrm{T}}\left(\mathrm{mV} /{ }^{\circ} \mathrm{C}\right)$, and (b) - the minimum $\mathrm{TC}$ point (MTC, referred to as ZTC (zero TC) in some sources [5]). The MTC point represents the value of the drain current at which the magnitude of the $\mathrm{V}_{\mathrm{T}} \mathrm{TC}$ is minimised $(\mathrm{mA})$.
The $I-V$ curves were measured at temperatures from 40 to $100^{\circ} \mathrm{C}$, with increments of $20^{\circ} \mathrm{C}$. The gate voltage $\mathrm{TC}$ representing the $\mathrm{V}_{\mathrm{T}} \mathrm{TC}$ is plotted as the red line; the red markers show the size of the drain current steps. The MTC point is displayed at the zero value of the TC curve magnitude.the MTC point current is also equal to the drain current at which the $I-V$ curves cross each other.

The markers on the dut17 curve show the total dose resolution of the measurements. this chart was plotted with $53 I-V$ curve data sets.

The markers on the dut17 curve show the total dose resolution of the measurements.the results are limited by the duts drain current range of $35 \mathrm{~mA}$.

The TID-induced changes in the $V_{\mathrm{T}} \mathrm{TC}$ were determined at the drain current of $1 \mathrm{~mA}$ by using 53 measured $I-V$ curve data sets. The resulting traces of the DUTs (selected as the most sensitive ones) in Fig. 11 exhibit a significant gate bias voltage dependence as well as an initial reversed polarity of the $V_{\mathrm{T}}$ TC. The TID-driven changes of the MTC points were extracted from the same data via interpolation of the two lowest points of the TC as a function of the drain current. The MTC measurements show similar effects on the $\mathrm{V}_{\mathrm{T}} \mathrm{TC}$ (Fig. 12).

\section{Voltage reference TID-TC experiment}

The second TID-TC experiment within this paper was focused on TID-induced changes in various commercial voltage references [9]. These devices usually find 


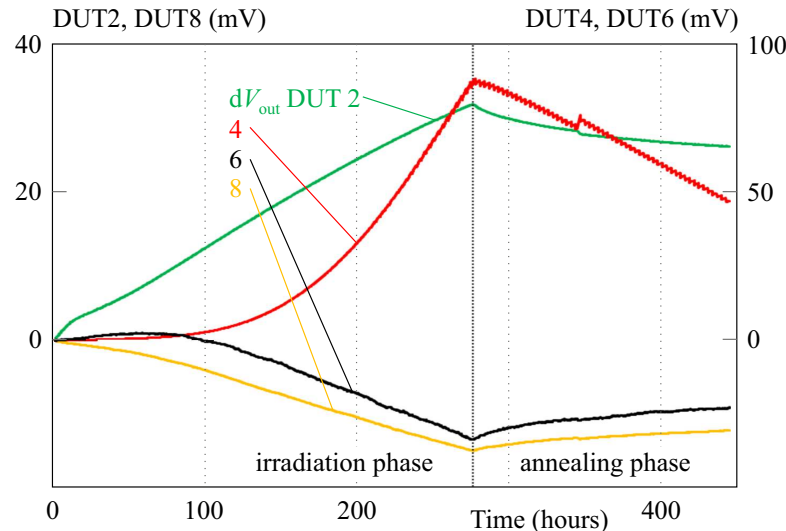

Fig. 14. The output voltage degradation and recovery in the biased DUTs during the irradiation and annealing

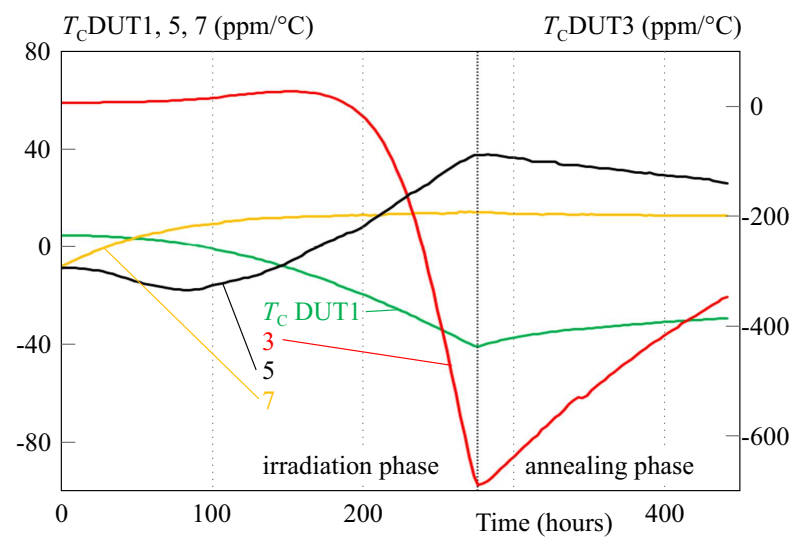

Fig. 16. The change and recovery of the temperature coefficients of the unbiased DUTs during the irradiation and annealing

use in high-resolution data acquisition systems. A set of eight commercial voltage references (DUT1 to DUT8) was used, with four different voltage reference types [2326 ] and two identical devices of each type, as defined in Tab. 1. The two DUTs invariably originated from the same production lot, similarly to the scheme of the initial TID experiment [27]. In each type, the first DUT was not biased in the course of irradiation (the VIN pin was grounded), with the bias voltage applied only during the electrical measurement. The second DUT was permanently biased.

In the voltage reference tests, the measurement system (MS) was an updated version of the setup employed in the initial experiment [27]. The MS was fully automated and comprised a DUT container placed in an irradiation chamber, test instrumentation, and a $\mathrm{PC}$. The instruments were positioned outside the irradiation facility.

In order to measure the output voltage of the DUTs, we used a high-resolution, 7.5 digit digital multimeter (a Keithley DMM7510). The device was set to provide the highest possible resolution, using 10 NPLC (number of power line cycles) to suppress the power line noise.

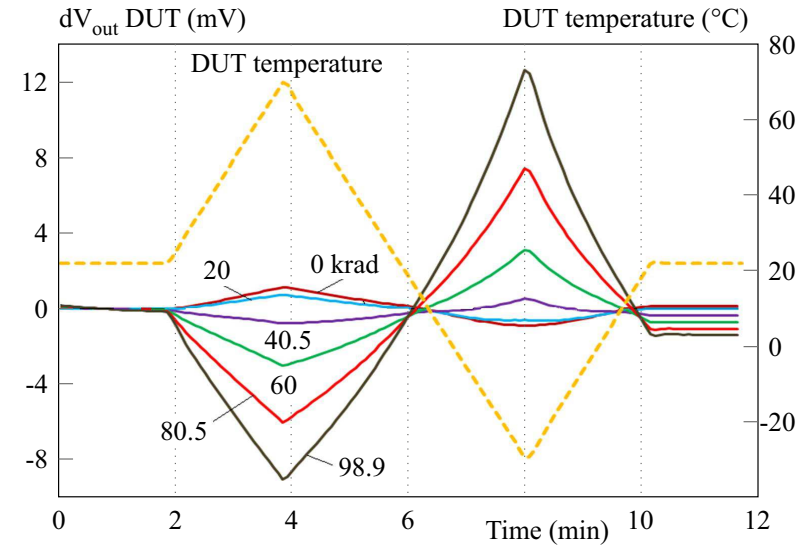

Fig. 15. The TID-induced change of the DUT1 response to the temperature sweep cycle

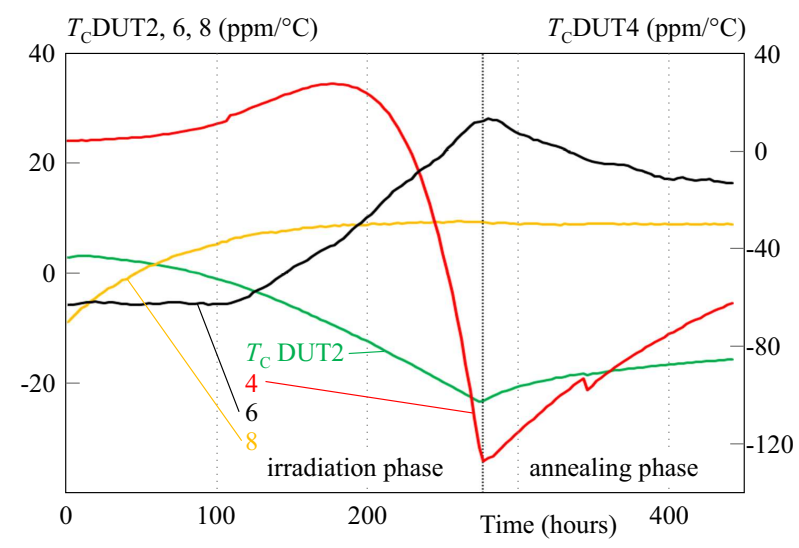

Fig. 17. The change and recovery of the temperature coefficients of the biased DUTs during the irradiation and annealing

The multiplexing of the DMM7510 input was ensured via CMOS analogue multiplexors. The DUTs were connected by $15 \mathrm{~m}$ long shielded twisted cables.

Regular measurements of the DUT output voltage were performed, either at the idle temperature of $22^{\circ} \mathrm{C}$ or during a ramp-style temperature sweep. During the idle temperature cycles, the DMM7510 was configured to yield the highest resolution and accuracy by enabling auto-zeroing, line synchronisation, and averaging. The idle temperature measurements were executed every 15 minutes. The temperature sweep exhibited a saw profile, and the trend of $0.4^{\circ} \mathrm{C} / \mathrm{s}$ facilitated fast measurement and sweep time reduction to less than 12 minutes. The cycle was performed every 3 hours, correspondingly to the cumulated dose of $1.08 \mathrm{krad}(\mathrm{Si})$ per sweep. The dose rate of $360 \mathrm{rad}(\mathrm{Si}) / \mathrm{hr}(100 \mathrm{mrad}(\mathrm{Si}) / \mathrm{sec})$, produced by the cobalt 60 source, was used.

The voltage reference experiment started with installing the test system in the irradiation facility and running pre-irradiation measurements to ensure stabilisation. The second phase consisted in irradiation up to the TID of $100 \mathrm{krad}(\mathrm{Si})$, followed by 70 hours of annealing. The third phase then involved long-term annealing, 
during which the entire experiment was arranged in an electronics laboratory. The radiation-induced changes in the output voltage of the unbiased DUTs are plotted in Fig. 13; the same record for the biased devices is displayed in Fig. 14. A significant bias sensitivity becomes obvious when the scales of the charts are compared. The glitches on the DUT2 and DUT4 annealing curves were caused by the absence of biasing during relocation of the test system to the electronics laboratory.

The chart in Fig.15 shows how the TID gradually changed the response of the DUT1 to the temperature sweep. Only selected temperature sweeps at TID steps of $20 \mathrm{krad}(\mathrm{Si})$ are displayed. The temperature sweep data were processed to yield the temperature coefficients (TCs). The TCs of each DUT were calculated by using linear regression in the course of the TC sweep cooling stage between +70 and $-30{ }^{\circ} \mathrm{C}$. The resulting changes in the TCs can be seen in Fig. 16 and Fig.17.

\section{Discussion}

The PMOS experiment provided the first opportunity to employ the proposed method in practice, namely, for the in-situ measurement of TID-induced TC changes (TID-TC) by using the novel DTC system. After several minor problems had been solved, the system became a precise and reliable tool to control the DUT temperature. During the experiments the test software not only experimental data, but it was also collecting and analysing various engineering data from the DTC system. The DTC did not exhibit any performance anomaly in the course of the more than 100 operating days (with nearly 44 million DUT temperature control tasks performed), and no TIDinduced degradation was observed in any primary part of the DTC system placed in the irradiation container. This excellent performance record validated the design of the DTC system and its readiness for advanced TID-TC experiments.

Without a database on the ZVP transistor series and given that structure was not published, the results of the PMOS experiment proved rather difficult to confirm; the outcomes, however, can be compared with data obtained previously from RADFETs [3-5].

As expected, all the measured parameters exhibited strong dependence on the gate-bias conditions. There was good agreement between the observed threshold voltage shift bias dependence and the irradiation curves represented in [5], Fig. 2. The significant difference in the DUT-DUT responsivity cannot be linked to individual structures of the DUTs: No mask data were accessible, and we did not know if the DUTs had come from the same wafer.

The initial reversed polarity of the TC and MTC curves was not expected and requires more detailed (statistical) analysis. Such behaviour does not appear in the unbiased DUTs. Another, and rather surprising, result consisted in the positive temperature dependence of the $\Delta V_{\mathrm{T}}$ ripple observed in all the DUTs; this effect does not seem to be caused by the real time thermal annealing, which was assumed to have a negative temperature dependence.

The data from the voltage reference experiment suggest excellent functionality of the test system designed for this project, showing that a measurement resolution above $10 \mu \mathrm{V}$ is achievable even with the measuring setup placed outside the irradiation facility and regardless of the complex, $15 \mathrm{~m}$ long cabling.

The observed TID-induced degradation of the output voltage of the DUTs was compared with the outcomes of the previous experiment [27], as the DUTs originated from the same production lots and the measurements were conducted under equivalent conditions. The resulting curves exhibited identical shapes (Fig. 14 and Fig. 15), but the magnitude of the degradation was markedly lower during the experiment (with the maximum degradation at about $30 \%$ and $50 \%$ of the previous experimental values established in the unbiased and biased DUTs, respectively). This discrepancy could be explained by the thermal annealing in the temperature sweeps, which reduced the overall degradation. In general, the DUT output voltage does not fully return to the initial value at the end of the temperature sweep cycle (Fig. 16); such a condition is caused by the thermal hysteresis of the DUTs and becomes more significant with increasing dose. This effect can be also observed as a ripple on the degradation plots (Fig. 14 and Fig. 15).

The initial pre-irradiation readings of the temperature coefficients were in good agreement with the datasheet specifications. Most of the readings varied within the window of $\pm 0.2 \mathrm{ppm} /{ }^{\circ} \mathrm{C}$, indicating excellent stability and repeatability of the measurement method. The TID changes of the temperature coefficients were rather complex, as can be seen in Fig. 16 and Fig. 17; however, they shared one feature, namely, the initial polarity of the TC changing with the TID. This common aspect points to an initial TID window during which the TC gradually decreased to be virtually zero in certain TIDs. A similar effect was observed in the PMOS transistors (Fig. 11 and Fig. 12). The TCs of DUT7 and DUT8 showed saturation at $50 \mathrm{krad}$, with the TC having opposite polarities but also values similar to that of the starting TC. All the other DUTs continued to change the TCs significantly. The TC of DUT3 constituted the worst case, having increased by more than two orders of magnitude. A similar result was obtained from DUT1, in agreement with source [28], where comparable data had been reported.

\section{Conclusion}

This paper presents the results of comprehensive experiments designed to examine TID changes of temperature coefficients in two types of COTS devices by using a novel TID-TC test method. To support the relevant procedures, a sophisticated temperature control system was developed and tested. 
The presented PMOS data clearly show radiationinduced trends in the TCs of the measured parameters. The innovative approach to controlling the temperature of the DUTs, together with the fine total dose resolution, enabled us to observe certain new effects, such as unexpected polarity of the thermally induced ripple on the threshold voltage shift, initial polarity change in the MTC point shift, and potential bias voltage saturation of the threshold voltage shift temperature coefficient.

The results of the voltage reference experiment suggest that, in general, the temperature coefficients change significantly with the TID, but within an initial limited TID window the temperature coefficients are lowered and thus the devices are, in fact, less sensitive to the variation of ambient temperature on-board a spacecraft.

\section{Acknowledgements}

The presented research was financed by the Czech Ministry of Education within the National Sustainability Program, grant LO1401 INWITE. For the research, infrastructure of the SIX Centre was used.

\section{REFERENCES}

[1] T. P. Ma and P. V. Dressendorfer, "Ionizing radiation effects in MOS devices circuits", 1/E, New York: Wiley-Interscience, 1989.

[2] A. Holmes-Siedle and L. Adams, "Handbook of radiation effects", 2/E, Oxford: Oxford University Press, 2002.

[3] A. Holmes-Siedle, F. Ravotti, and M. Glaser, "The dosimetric performance of RADFETs in radiation test beams", Proceedings of IEEE Radiation Effects Data Workshop, pp. 42-57.

[4] G. Sarrabayrouse and S. Siskos, "Behaviour of High Sensitivity MOS Radiation Dosimeters Biased in the MTC Current Region", Proceedings of the 9th WSEAS Int. Conference on Instrumentation Measurement, Circuits Systems, pp. 38-41, 2010.

[5] A. Haran, A. Jaksic, N. Refach, A. Eliyahu, D. David, and J. Barak, "Temperature Effects Long Term Fading of Implanted Un-Implanted Gate Oxide RADFETs", Proceedings of the 7th European Conference on Radiation and Its Effects on Components Systems (RADECS), pp. 465-469, 2003.

[6] J. Hofman and R. Sharp, "Measurement Methods for Total Ionising Dose Testing: In-Situ versus Standard Practice", Proceedings of IEEE Radiation Effects Data Workshop, pp. 1-4, 2012.

[7] J. Hofman, A. Holmes-Siedle, R. Sharp, and J. Haze, "A Method for In-situ Total Ionising Dose Measurement of Temperature Coefficients of Semiconductor Device Parameters", IEEE Trans, Nucl. Sci. vol. 62, no. 6, pp. 2525-2531, Dec 2015.

[8] J. Hofman, A. Jaksic, R. Sharp, N. Vasovic, and J. Haze, "In-Situ Measurement of Total Ionising Dose Induced Changes in Threshold Voltage Temperature Coefficients of RADFETs", IEEE Trans, Nucl, Sci. vol. 64, no. 1, pp. 582-586, 2017.
[9] J. Hofman, R. Sharp, and J. Haze, "TID In-Situ Measurement of Temperature Coefficient of Various Commercial Voltage References", Proceedings of 17th European Conference on Radiation and Its Effects on Components Systems (RADECS), pp. 1-4, 2017.

[10] Temperature Test Chambers TempEvent Brochure, 3/E, Balingen: Weiss Umwelttechnik 2017.

[11] Test method standard microcircuits MIL-STD-883J TM 1019.9, 9/E, Washington DC: US Department of Defence, 2013.

[12] ThermalAir TA-5000 Brochure, 1/E, New York: MPI Thermal, 2017.

13] D. M. Rowe, "CRC Handbook of Thermoelectrics", 1/E, Boca Raton: CRC Press 1995.

[14] J. Scholz and T. Ricolfi, "Sensors, A Comprehensive Survey", vol. 4 Thermal Sensors, 2/E, New York: VCH Publishers, 2008.

[15] M. Gonzlez-Guerrero, M. T. Álvarez, J. J. Jiménez, J. Sánchez -Páramo, and H. Guerrero, "The effect of proton gamma radiation on different types of temperature sensors", Proceedings of European Conference on Radiation and Its Effects on Components Systems (RADECS), pp. 41-45, 2009.

[16] ADS1248 24-Bit Analog-to-Digital Converters for Temperature Sensors, 2/E, Dallas: Texas Intruments, 2011.

[17] J. Hofman, "In-situ, low dose rate, total ionising dose test of the cooling performance of a thermoelectric module", Proceedings of 14th European Conference on Radiation and Its Effects on Components Systems (RADECS), pp. 1-4, 2013.

[18] P. Balda and M. Schlegel, "Advanced PID Control Algorithms Built into the REX Control System", IFAC Proc. vol. 45, no. 3 , pp. 465-470, 2012.

[19] J. Hofman, "Photovoltaic Panels Efficiency Measurement System", Master thesis 1/E, Brno: BUT Department of Microlelectronics, 2010.

[20] K. Instruments and A. Road, "Model DMM7510 7-1/2 Digit Gq raphical Sampling Multimeter Specifications", pp. 1-28, Nov. q 2014.

[21] "User's manual FLIR T650 series", 2/E, New York: FLIR Systems, 2017.

[22] "ZVP1320F Datasheet", 4/E, Plano: Diodes Incorporated, 2012.

[23] "LT1236 Precision Reference", 1/E, Milpitas: Linear Technology, 1995.

[24] "ADR440 Ultralow Noise, LDO XFET Voltage References with Current Sink and Source", 5/E, Norwood: Analog Devices, 2010.

[25] "LT1460 Micropower Precision Series Reference Family", 3/E, Milpitas: Linear Technology, 2006.

[26] "ADR03 Ultracompact, Precision 2.5 V Voltage Reference", 18/E, Norwood: Analog Devices, 2012,.

[27] J. Hofman, R. Sharp, and J. Haze, "In-Situ Measurement of Total Ionising Dose Induced Degradation of Various Commercial Voltage References", in Proceedings of 16th European Conference on Radiation Its Effects on Components Systems (RADECS), pp. 1-4, 2016.

[28] "LM4050QML Precision Micropower Shunt Voltage Reference", $7 / \mathrm{E}$, Texas Instruments, 2013.

Received 2 March 2019 